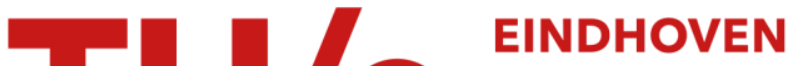 \\ UNIVERSITY OF \\ TECHNOLOGY
}

\section{Inhomogeneous critical current in nanowire superconducting single-photon detectors}

Citation for published version (APA):

Gaudio, R., Hoog, op 't, K. P. M., Zhou, Z., Sahin, D., \& Fiore, A. (2014). Inhomogeneous critical current in nanowire superconducting single-photon detectors. Applied Physics Letters, 105, 1-4. [222602].

https://doi.org/10.1063/1.4903071

DOI:

10.1063/1.4903071

Document status and date:

Published: 01/01/2014

\section{Document Version:}

Publisher's PDF, also known as Version of Record (includes final page, issue and volume numbers)

\section{Please check the document version of this publication:}

- A submitted manuscript is the version of the article upon submission and before peer-review. There can be important differences between the submitted version and the official published version of record. People interested in the research are advised to contact the author for the final version of the publication, or visit the $\mathrm{DOI}$ to the publisher's website.

- The final author version and the galley proof are versions of the publication after peer review.

- The final published version features the final layout of the paper including the volume, issue and page numbers.

Link to publication

\section{General rights}

Copyright and moral rights for the publications made accessible in the public portal are retained by the authors and/or other copyright owners and it is a condition of accessing publications that users recognise and abide by the legal requirements associated with these rights.

- Users may download and print one copy of any publication from the public portal for the purpose of private study or research.

- You may not further distribute the material or use it for any profit-making activity or commercial gain

- You may freely distribute the URL identifying the publication in the public portal.

If the publication is distributed under the terms of Article 25fa of the Dutch Copyright Act, indicated by the "Taverne" license above, please follow below link for the End User Agreement:

www.tue.nl/taverne

Take down policy

If you believe that this document breaches copyright please contact us at:

openaccess@tue.nl

providing details and we will investigate your claim. 


\title{
Inhomogeneous critical current in nanowire superconducting single-photon detectors
}

\author{
R. Gaudio, ${ }^{\text {a) }}$ K. P. M. op 't Hoog, Z. Zhou, D. Sahin, ${ }^{\text {b) }}$ and A. Fiore \\ COBRA Research Institute, Eindhoven University of Technology, P.O. Box 513, NL-5600MB Eindhoven, \\ The Netherlands
}

(Received 7 October 2014; accepted 18 November 2014; published online 1 December 2014)

\begin{abstract}
A superconducting thin film with uniform properties is the key to realize nanowire superconducting single-photon detectors (SSPDs) with high performance and high yield. To investigate the uniformity of $\mathrm{NbN}$ films, we introduce and characterize simple detectors consisting of short nanowires with length ranging from $100 \mathrm{~nm}$ to $15 \mu \mathrm{m}$. Our nanowires, contrary to meander SSPDs, allow probing the homogeneity of $\mathrm{NbN}$ at the nanoscale. Experimental results, endorsed by a microscopic model, show the strongly inhomogeneous nature of $\mathrm{NbN}$ films on the sub-100 $\mathrm{nm}$ scale. (C) 2014 AIP Publishing LLC. [http://dx.doi.org/10.1063/1.4903071]
\end{abstract}

Superconducting single-photon detectors (SSPDs) $^{1,2}$ based on niobium nitride $(\mathrm{NbN})$ nanowires present high speed, low dark counts, and low jitter. ${ }^{3,4}$ Compared to more efficient SSPDs recently obtained from amorphous superconducting materials, ${ }^{5-9} \mathrm{NbN}$ SSPDs allow operation at higher temperatures and with simpler read-out circuitry. Due to such favourable combination, SSPDs have brought a breakthrough in fields such as quantum key distribution (QKD), ${ }^{10,11}$ nanoscale imaging, ${ }^{12}$ and quantum optics. ${ }^{13}$

However, the number of possible applications is still limited by the low fabrication yield. Indeed, realizing arrays of SSPDs with the same performance is challenging. Understanding and solving this issue could enable free-space single-photon imaging, ${ }^{14}$ spatial and photon-number resolution, ${ }^{15-17}$ as well as circumventing dead time limitations in interplanetary optical communication. ${ }^{18}$

The physical reason for the poor reproducibility must be sought in the SSPD operating principle: for efficient operation, NbN SSPDs must be biased with a bias current $\left(I_{b}\right)$ slightly lower than the critical current $\left(I_{c}\right)$. In this condition, the diffusion of photocreated quasiparticles, together with the vortex unbinding, results in a voltage pulse in the readout circuit. ${ }^{19}$ This requires the nanowire to be extremely homogeneous, so that the critical current is uniform along its entire length.

Recent studies ${ }^{20,21}$ on meander SSPDs showed a variation in detection efficiency among nominally identical devices. The variation has been ascribed to highly localized areas of the nanowire, named constrictions, characterized by a reduced cross section. The large active area of the meander SSPD used in those studies, though, does not allow a straightforward investigation of such defects. Indeed, the dimensions, density, and physical origin of these constrictions are still unknown. In order to address these questions, here we investigate simpler detectors whose active areas consist of short wires with different lengths (L) and fixed

\footnotetext{
a)r.gaudio@tue.nl

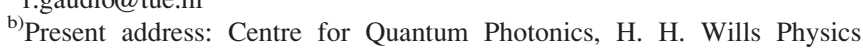
Laboratory, University of Bristol, Tyndall Avenue, Bristol BS8 1TL, United Kingdom
}

width $(\mathrm{w}=100 \mathrm{~nm})$. With these simple devices, not only we unequivocally prove that the critical current is not uniform but also we investigate the typical length scale of its variations. Our experimental results, endorsed by a microscopic model, reveal that the nanowire is not affected by isolated pointlike constrictions but is rather continuously inhomogeneous and the typical correlation length of the critical current variation is shorter than $100 \mathrm{~nm}$.

The fabrication process starts with the deposition of a 5-nm-thick $\mathrm{NbN}$ film on a GaAs (001) substrate by means of $\mathrm{DC}$ reactive magnetron sputtering. The $\mathrm{NbN}$ film is deposited by sputtering a $\mathrm{Nb}$ target in $\mathrm{Ar}+\mathrm{N}_{2}$ mixture at total pressure $P_{T O T}=2.3 \mathrm{mTorr}$. The deposition is carried out at a nominal temperature of $400{ }^{\circ} \mathrm{C}$, with target current of $250 \mathrm{~mA}$ and target voltage of $380 \mathrm{~V}$. These deposition conditions, similar to those previously used to fabricate highperformance meander- and waveguide-SSPDs, ${ }^{22-24}$ allowed the realization of a film with critical temperature $T_{c}=9.67 \mathrm{~K}$ and transition width $\Delta T_{c}=0.34 \mathrm{~K}$. Then contact pads, consisting of $14 \mathrm{~nm} \mathrm{Ti}$ and $140 \mathrm{~nm}$ Au layers, are defined through optical lithography, electron beam evaporation and lift off. In the final step, the nanowires are patterned from the $\mathrm{NbN}$ film by electron-beam lithography and reactive-ion etching in $\mathrm{Ar} / \mathrm{SF}_{6}$ plasma. The process was optimized in order to fabricate $100 \mathrm{~nm}$ wide nanowires with lengths ranging from $100 \mathrm{~nm}$ to $15 \mu \mathrm{m}$. In order to prevent latching, ${ }^{25}$ an additional meander (500 nm wide and $573 \mu \mathrm{m}$ long) is defined together with the nanowire to provide a series inductance of $103 \mathrm{nH}^{26}$ Figure 1 shows the scanning electron microscope (SEM) images of two nanowires with different lengths.

The electro-optical characterization is performed at an estimated sample temperature of $5 \mathrm{~K}$ in a cryogenic probe station with an optical window. During the electrical measurements, the detector is biased through the DC port of a bias-T with a voltage source connected in series with a $10 \Omega$ bias resistor. The RF port of the bias-T is connected to a $50 \Omega$ cap. The electrical contact with the device is established with a $50 \Omega$ microwave probe connected to the circuit through an SMA coaxial cable. A chip containing 16 nominally identical nanowires for each length $\mathrm{L}$, with 


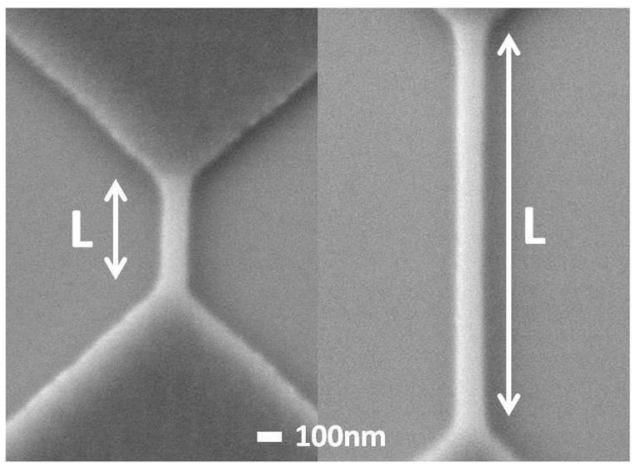

FIG. 1. SEM micrographs of a $100 \mathrm{~nm}$ wide nanowire with length $\mathrm{L}=400 \mathrm{~nm}$ (left) and $\mathrm{L}=1600 \mathrm{~nm}$ (right).

$\mathrm{L}=100 \mathrm{~nm}, 400 \mathrm{~nm}, 1.6 \mu \mathrm{m}$, and $15 \mu \mathrm{m}$, is measured in a single cooling run. While it limits the statistics, this procedure ensures that all the devices are characterized at the same effective temperature. The measured critical currents $\left(I_{c}\right)$ are displayed in Figure 2 as a function of nanowire length. The data, covering more than two orders of magnitude in wire length, show a clear trend. The critical current, ideally independent of wire length, decreases with increasing length. In addition, the $I_{c}$ values present a large spread, as measured by the standard deviation $\Delta I_{c}$, even for the shortest wires, for which the spread is maximum. To highlight the previous observations, we reported the same set of data in the histogram of Figure 3 (panel a).

The variation of $I_{c}$ values among nominally identical devices constitutes a direct proof for the inhomogeneous nature of the wire. A more intriguing feature is the decrease in the $\left(\bar{I}_{c}\right)$ encountered already between $100 \mathrm{~nm}$ and $400 \mathrm{~nm}$ long nanowires. These observations reveal that each nanowire presents a continuously inhomogeneous distribution of $I_{c}$ with correlation length shorter than or equal to $100 \mathrm{~nm}$. Indeed, if the nanowire was homogeneous on a $400 \mathrm{~nm}$ length scale, for example, we would not observe any decrease in the average $I_{c}$ between these and the $100 \mathrm{~nm}$ long

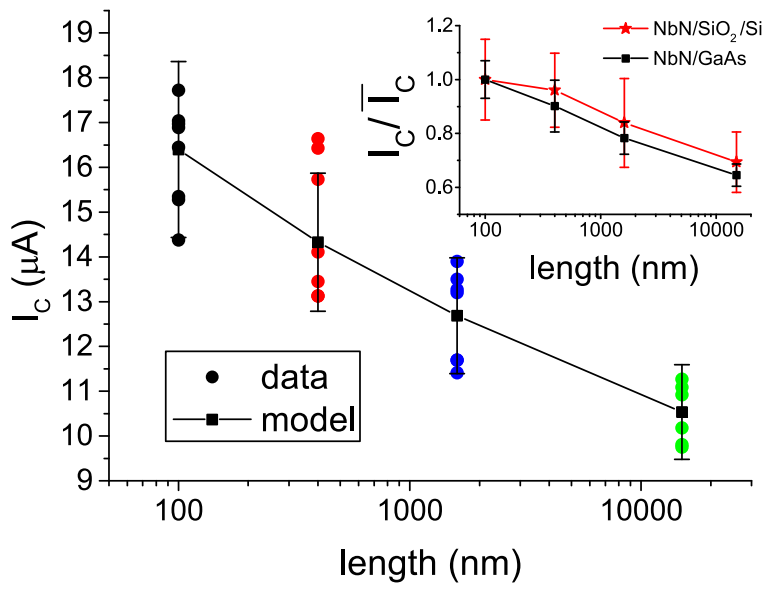

FIG. 2. Experimental values of critical currents displayed as a function of nanowire length (filled circles) are superimposed to the $\bar{I}_{c}$ (black squares) and standard deviation (error bars) calculated with the microscopic model. Inset: The data of the main panel is reported together with the data obtained from a commercially available $\mathrm{NbN}$ film. For a clear comparison, both sets of data are normalized to their mean $I_{c}\left(\bar{I}_{c}\right)$ and the standard deviation $\Delta I_{c}$ is calculated (error bars).

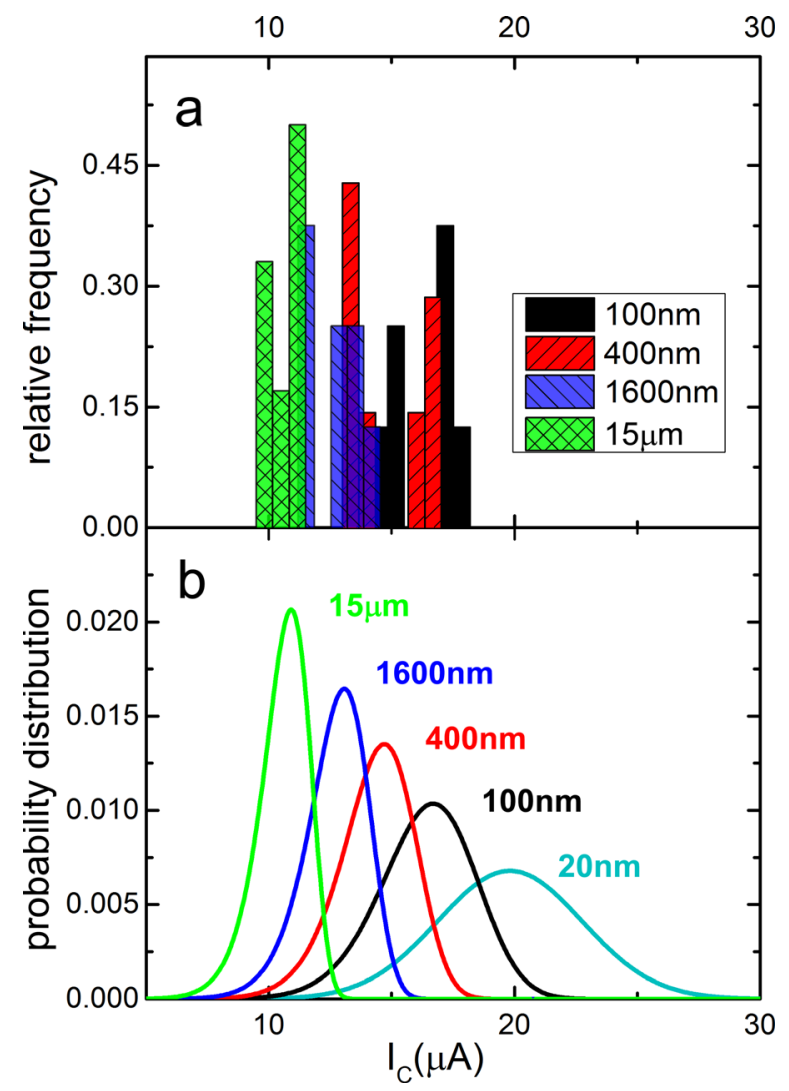

FIG. 3. The experimental $I_{c}$ histograms (panel a) are compared to the $I_{c}$ probability distribution calculated from the model (panel b). The light blue curve in panel $\mathrm{b}$ is the calculated probability distribution for the $20 \mathrm{~nm}$ section.

wires. Very similar results were obtained on different deposition batches of $\mathrm{NbN} / \mathrm{GaAs}$ films at different deposition temperatures. In a separate batch, a decrease in the $\bar{I}_{c}$ has been observed also between $\mathrm{L}=0 \mathrm{~nm}$ (bowtie detector ${ }^{12}$ ) and $\mathrm{L}=100 \mathrm{~nm}$ nanowires, indicating that the correlation length is smaller than $100 \mathrm{~nm}$. In order to understand whether this behaviour is only typical of our $\mathrm{NbN}$ films on GaAs, we realized and characterized the same detectors on a commercial $\mathrm{NbN}$ film deposited by SCONTEL Superconducting Nanotechnology on a $(250 \mathrm{~nm}) \mathrm{SiO}_{2} / \mathrm{Si}(001)$ substrate. The $\mathrm{NbN}$ film had a critical temperature of $T_{c}=10.03 \mathrm{~K}$. Since the detectors patterned from this film were measured at a different temperature $(\sim 2 \mathrm{~K})$, a straightforward comparison with the results of Figure 2 (main panel) is only possible with normalized data. In the inset of Figure 2, we compare the $\bar{I}_{c}$ and $\Delta I_{c}$ values obtained from the two samples where both sets of data are normalized to the $\bar{I}_{c}$ of their $100 \mathrm{~nm}$ long wires. The graph clearly shows that the reported behaviours are not unique to our $\mathrm{NbN}$ films. In fact, also detectors patterned from the $\mathrm{NbN} / \mathrm{SiO}_{2}$ film are characterized by the decrease of $\bar{I}_{c}$ with increasing length, and they have a large spread in critical current $\Delta I_{c}$ for each length. It is worth noticing that in both samples the ratio between the normalized $\bar{I}_{c}$ of the longest and shortest wires is almost 0.6. While we cannot exclude that more homogeneous films may be obtained by varying the deposition conditions, as the results in Ref. 19 suggest the observed inhomogeneity is likely typical of NbN films used for SSPD fabrication. To gain more insight, we calculated the theoretical depairing current $\left(I_{c}^{\text {dep }}\right)$ 
and compared it with the $I_{c}$ values resulted from our measurements. The depairing current is estimated according to the Ginzburg-Landau model and the temperature dependence proposed by Bardeen. ${ }^{27,28}$ In the $I_{c}^{\text {dep }}$ estimation, we considered the following quantities: the measured sheet resistance in the normal state $R_{s}=870 \Omega / \mathrm{sq}$, the diffusivity $\mathrm{D} \approx 0.5 \mathrm{~cm}^{2} / \mathrm{s}$ of NbN films, ${ }^{29}$ the measured critical temperature $T_{c}=9.67 \mathrm{~K}$ and the energy gap at zero temperature $\Delta(0)=2.07 \mathrm{k}_{\mathrm{B}} \mathrm{T}_{\mathrm{c}}{ }^{29}{ }^{2}$ For a $100 \mathrm{~nm}$ wide nanowire operating at $5 \mathrm{~K}, I_{c}^{d e p} \sim 21 \mu \mathrm{A}$. We note that the $I_{c}$ of the long wires is almost half of the calculated $I_{c}^{d e p}$ as previously observed. ${ }^{28,30}$ Our results therefore suggest that the inhomogeneity is the main reason for the low observed critical currents in long meanders.

We developed a simple microscopic model to endorse our experimental observations. The nanowire is modelled as a chain of identical sections (width $100 \mathrm{~nm}$ and length s) each of which has a different critical current $I_{c i}$. We assume that the probability for a section to have a certain $I_{c i}$ value is given by a Gaussian probability distribution with mean value $\mu$ and standard deviation $\sigma$. For a wire of $\mathrm{N}$ sections $(\mathrm{N}=\mathrm{L} / \mathrm{s})$ the critical current $I_{c \text {,wire }}$ will be given by the minimum $I_{c i}$ among the $\mathrm{N}$ different values. For each wire length and for a given $(\mu, \sigma)$, we calculate the probability that the entire chain of sections has the critical current $i_{c i}$, where $0<i_{c i}<50 \mu \mathrm{A}$. The model starts with an initial guess for $(\mu, \sigma)$ and proceeds by iterating the probability calculation for different $(\mu, \sigma)$. The model stops when the pair $(\mu, \sigma)$ minimizing the $\chi^{2}$ between the modelled and experimental $\bar{I}_{c}$ is found, assuming a section length $\mathrm{s}=20 \mathrm{~nm}$. The best agreement for the four wire lengths is found for $\mu=19.82 \mu \mathrm{A}$ and $\sigma=2.94 \mu \mathrm{A}$. The calculated $I_{c}$ probability distributions (for $I_{c}$ intervals of $0.2 \mu \mathrm{A}$ ) are reported in Figure 3 (panel b) together with the Gaussian distribution for the $I_{c}$ of the $20 \mathrm{~nm}$ section. This graph, when compared to the histograms of panel a, clearly shows that the decrease in both $\bar{I}_{c}$ and $\sigma$ is reproduced quantitatively by this simple model. In addition, in Figure 2 the average $I_{c}$ values and the standard deviations predicted by the model are superimposed to the experimental results. The agreement further proves that with simple assumptions we can quantitatively describe the electrical behaviour of our devices. We note that a comparable agreement can be found with any section length $\mathrm{s} \leq 100 \mathrm{~nm}$, so that the present data does not allow a more precise estimation of the correlation length. It should be noted that the best estimate of the average critical current for the $20 \mathrm{~nm}$ section is very close to the theoretical depairing current, which further supports our interpretation. In our search for the cause of this inhomogeneity, we extensively investigated SEM micrographs of our nanowires. Since the width, measured with the image processing software $\mathrm{WSxM},{ }^{32}$ has a standard deviation of $2.7 \%$, smaller than the relative variation in the $I_{c}: \Delta I_{c} / \bar{I}_{c} \sim 6.1 \%$, we tentatively attribute the inhomogeneity to the variation of film thickness and/or crystal properties.

Since a long wire can only be biased at almost half of the $\bar{I}_{c}$ of the shortest wire, we expect the internal quantum efficiency (QE) of SSPDs to be strongly limited. To extract more information about the effect of inhomogeneities on the efficiency, we studied the optical response of selected wires.
For each wire length, we selected 5-6 devices and measured them during two consecutive cooling runs. To ensure temperature reproducibility, we made sure that the measured $I_{c}$ values were the same within $1.2 \%$ in both cooling runs. During the optical characterization, a laser beam at $\lambda=1300 \mathrm{~nm}$ is focused onto a spot with full-width-half-maximum of $20 \mu \mathrm{m}$ to illuminate one detector at a time. With respect to the circuit used during the electrical characterization, the $50 \Omega$ cap is removed and a $4 \mathrm{~dB}$ attenuator connected to a series of four amplifiers (with $60 \mathrm{~dB}$ total amplification) is now added to the RF port of the bias-T. The detector output signal coming from the amplifiers is then sent to a counter. For each nanowire length, the light power is chosen in order to avoid multiphoton response and device heating. For this last purpose, the ratio between the device critical current measured with and without laser spot is always kept above 0.96 . In these conditions, we recorded detector counts with (photon counts, PC) and without (dark counts, DC) laser spot while varying the bias current, $I_{b}$. The QE is calculated by normalizing the difference between PC and DC by the number of photons impinging on the detector active area $(100 \mathrm{~nm} \times \mathrm{L})$. We note that this definition of $\mathrm{QE}$ slightly differs from the device $\mathrm{QE}$ used for meander detectors where the entire area of the device is considered.

The QE curves in Figure 4 (main panel) show that a detector responds to incoming light with its highest efficiency only when it is biased very close to its $I_{c}$. As a consequence of the $I_{c}$ decrease with increasing detector length, the QE curves of short nanowires always extend to higher $I_{b}$ and $\mathrm{QE}$ values with respect to the long nanowire case. The previous observation is made explicit by the inset of Figure 4 where the maximum quantum efficiency values are plotted as a function of the $I_{c}$. The inset clearly shows how a lower $I_{c}$ corresponds to a lower $\mathrm{QE} .{ }^{31}$ In addition, the curves of the main panel show that at fixed and low $I_{b}$ the long wires appear to be more efficient than the short ones. This is related to the fact that some sections of the long wires operate at a bias current closer to their critical current. However, when averaging over many devices, we would expect the same efficiency in long and short wires, since long wires effectively average over many short wires. It could result from the limited statistics available, whereby short wires

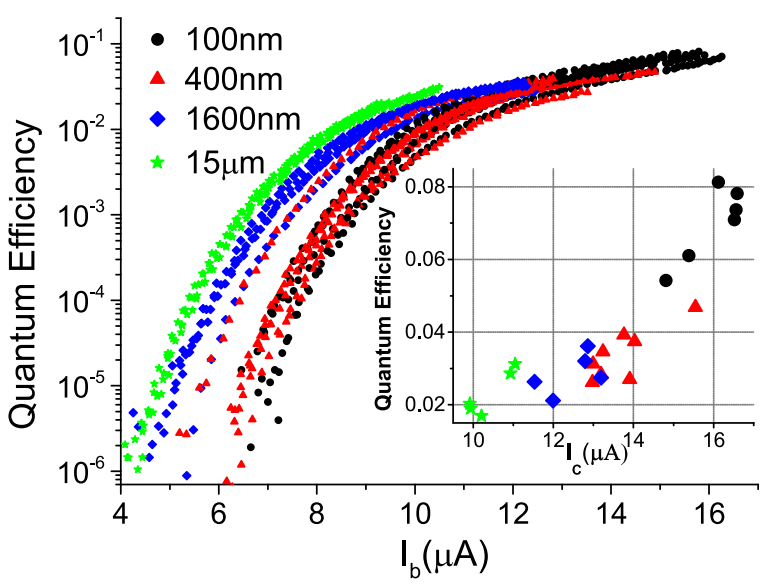

FIG. 4. Quantum efficiency curves for devices of different lengths are plotted as function of $I_{b}$. Inset: maximum quantum efficiency data is plotted as function of device $I_{c}$. 
with low $I_{c}$, and therefore much more efficient at low $I_{b}$, are not likely to be measured.

In summary, we have investigated the inhomogeneous nature of $\mathrm{NbN}$ nanowires by systematically studying the critical current and efficiency of wires with different lengths. The observed trends cannot be explained by the presence of isolated constrictions, but rather strongly indicate that the wires are inhomogeneous at a length scale shorter than or equal to $100 \mathrm{~nm}$. While more investigations are needed in order to determine the physical origin of the non-uniformity of the critical current, small fluctuations in the $\mathrm{NbN}$ film thickness being a likely candidate. We note that the proposed experimental method, based on the measurement of the critical current for a number of short wires, enables a simple and reproducible assessment of the film homogeneity and thereby provides more information than extensive QE measurements on long meanders.

The authors would like to thank Saeedeh Jahanmirinejad and Giulia Enrica Digeronimo for technical assistance, Sartoon Fattah poor for support with MATLAB code and Jelmer Renema, Michiel de Dood, and Martin van Exter for useful discussions. The nanofabrication was carried out in the Nanolab at TU/e cleanroom facility. This work is part of the research programme of the Foundation for Fundamental Research on Matter (FOM), which was financially supported by the Netherlands Organisation for Scientific Research (NWO), was also supported by NanoNextNL, a micro- and nanotechnology program of the Dutch Ministry of Economic Affairs, Agriculture and Innovation (EL \& I) and 130 partners and by the Dutch Technology Foundation STW, Applied Science Division of NWO, the Technology Program of the Ministry of Economic Affairs.

${ }^{1}$ G. Goltsman, O. Okunev, G. Chulkova, A. Lipatov, A. Semenov, K. Smirnov, B. Voronov, A. Dzardanov, C. Williams, and R. Sobolewski, Appl. Phys. Lett. 79, 705 (2001).

${ }^{2}$ C. M. Natarajan, M. G. Tanner, and R. H. Hadfield, Supercond. Sci. Technol. 25, 063001 (2012).

${ }^{3}$ A. Pearlman, A. Cross, W. Sysz, J. Zhang, A. Verevkin, M. Currie, A. Korneev, P. Kouminov, K. Smirnov, B. Voronov, G. Goltsman, and R. Sobolewski, IEEE Trans. Appl. Supercond. 15, 579 (2005).

${ }^{4}$ T. Yamashita, S. Miki, K. Makise, W. Qiu, H. Terai, M. Fujiwara, M. Sasaki, and Z. Wang, Appl. Phys. Lett. 99, 161105 (2011).

${ }^{5}$ B. Baek, A. E. Lita, V. Verma, and S. W. Nam, Appl. Phys. Lett. 98, 251105 (2011).

${ }^{6}$ V. B. Verma, F. Marsili, S. Harrington, A. E. Lita, R. P. Mirin, and S. W. Nam, Appl. Phys. Lett. 101, 251114 (2012).

${ }^{7}$ F. Marsili, V. B. Verma, J. A. Stern, S. Harrington, A. E. Lita, T. Gerrits, I. Vayshenker, B. Baek, M. D. Shaw, R. P. Mirin, and S. W. Nam, Nat. Photonics 7, 210 (2013).

${ }^{8}$ V. B. Verma, A. E. Lita, M. R. Vissers, F. Marsili, D. P. Pappas, R. P. Mirin, and S. W. Nam, Appl. Phys. Lett. 105, 022602 (2014).

${ }^{9}$ V. B. Verma, B. Korzh, F. Bussires, R. D. Horansky, A. E. Lita, F. Marsili, M. D. Shaw, H. Zbinden, R. P. Mirin, and S. W. Nam, Appl. Phys. Lett. 105, 122601 (2014).
${ }^{10}$ H. Takesue, S. W. Nam, Q. Zhang, R. H. Hadfield, T. Honjo, K. Tamaki, and Y. Yamamoto, Nat. Photonics 1, 343 (2007).

${ }^{11}$ D. Stucki, C. Barreiro, S. Fasel, J. Gautier, O. Gay, N. Gisin, R. Thew, Y. Thoma, P. Trinkler, F. Vannel, and H. Zbinden, Opt. Express 17, 13326 (2009).

${ }^{12}$ D. Bitauld, F. Marsili, A. Gaggero, F. Mattioli, R. Leoni, S. Jahanmiri Nejad, F. Levy, and A. Fiore, Nano Lett. 10, 2977 (2010).

${ }^{13}$ C. Zinoni, B. Alloing, L. H. Li, F. Marsili, A. Fiore, L. Lunghi, A. Gerardino, Y. B. Vakhtomin, K. V. Smirnov, and G. N. Goltsman, Appl. Phys. Lett. 91, 031106 (2007).

${ }^{14}$ M. Allman, V. B. Verma, R. Horansky, F. Marsili, J. A. Stern, M. D. Shaw, A. D. Beyer, R. P. Mirin, and S. W. Nam, "Progress towards a near IR single-photon superconducting nanowire camera for free-space imaging of light," in CLEO: 2014, OSA Technical Digest (online) (Optical Society of America, 2014), paper AW3P.3.

${ }^{15}$ A. Divochiy, F. Marsili, D. Bitauld, A. Gaggero, R. Leoni, F. Mattioli, A. Korneev, V. Seleznev, N. Kaurova, O. Minaeva, G. Goltsman, K. G. Lagoudakis, M. Benkhaoul, F. Levy, and A. Fiore, Nat. Photonics 2, 302 (2008).

${ }^{16}$ Z. Zhou, S. Jahanmirinejad, F. Mattioli, D. Sahin, G. Frucci, A. Gaggero, R. Leoni, and A. Fiore, Opt. Express 22, 3475-3489 (2014).

${ }^{17}$ V. B. Verma, R. Horansky, F. Marsili, J. A. Stern, M. D. Shaw, A. E. Lita, R. P. Mirin, and S. W. Nam, Appl. Phys. Lett. 104, 051115 (2014).

${ }^{18}$ M. Shaw, K. Birnbaum, M. Cheng, M. Srinivasan, K. Quirk, J. Kovalik, A. Biswas, A. D. Beyer, F. Marsili, V. Verma, R. P. Mirin, S. W. Nam, J. A. Stern, and W. H. Farr, "A Receiver for the lunar laser communication demonstration using the optical communications telescope laboratory," in CLEO: 2014, OSA Technical Digest (online) (Optical Society of America, 2014), SM4J.2.

${ }^{19}$ J. J. Renema, R. Gaudio, Q. Wang, Z. Zhou, A. Gaggero, F. Mattioli, R. Leoni, D. Sahin, M. J. A. de Dood, A. Fiore, and M. P. van Exter, Phys. Rev. Lett. 112, 117604 (2014)

${ }^{20}$ A. J. Kerman, E. A. Dauler, J. K. W. Yang, K. M. Rosfjord, V. Anant, K. K. Berggren, G. N. Goltsman, and B. Voronov, Appl. Phys. Lett. 90, 101110 (2007).

${ }^{21}$ R. H. Hadfield, P. A. Dalgarno, J. A. O'Connor, E. Ramsay, R. J. Warburton, E. J. Gansen, B. Baek, M. J. Stevens, R. P. Mirin, and S. W. Nam, Appl. Phys. Lett. 91, 241108 (2007).

${ }^{22}$ A. Gaggero, S. Jahanmiri Nejad, F. Marsili, F. Mattioli, R. Leoni, D. Bitauld, D. Sahin, G. J. Hamhuis, R. Nötzel, R. Sanjines, and A. Fiore, Appl. Phys. Lett. 97, 151108 (2010).

${ }^{23}$ J. P. Sprengers, A. Gaggero, D. Sahin, S. Jahanmiri Nejad, F. Mattioli, R. Leoni, J. Beetz, M. Lermer, M. Kamp, S. Höfling, R. Sanjines, and A. Fiore, Appl. Phys. Lett. 99, 181110 (2011).

${ }^{24}$ D. Sahin, A. Gaggero, Z. Zhou, S. Jahanmiri Nejad, F. Mattioli, R. Leoni, J. Beetz, M. Lermer, M. Kamp, S. Höfling, and A. Fiore, Appl. Phys. Lett. 103, 111116 (2013).

${ }^{25}$ A. J. Kerman, E. A. Dauler, W. E. Keicher, J. K. W. Yang, K. K. Berggren, G. N. Goltsman, and B. Voronov, Appl. Phys. Lett. 88, 111116 (2006).

${ }^{26}$ F. Marsili, D. Bitauld, A. Gaggero, S. Jahanmirinejad, R. Leoni, F. Mattioli, and A. Fiore, New J. Phys. 11, 045022 (2009).

${ }^{27}$ J. Bardeen, Rev. Mod. Phys. 34, 667 (1962).

${ }^{28}$ D. Henrich, P. Reichensperger, M. Hofherr, J. M. Meckbach, K. Il'in, M. Siegel, A. Semenov, A. Zotova, and D. Y. Vodolazov, Phys. Rev. B 86, 144504 (2012).

${ }^{29}$ A. Semenov, B. Gnther, U. Bttger, H. W. Hbers, H. Bartolf, A. Engel, A. Schilling, K. Ilin, M. Siegel, R. Schneider, D. Gerthsen, and N. A. Gippius, Phys. Rev. B 80, 054510 (2009).

${ }^{30}$ M. Hofherr, D. Rall, K. Ilin, M. Siegel, A. Semenov, H. W. Hbers, and N. A. Gippius, J. Appl. Phys. 108, 014507 (2010).

${ }^{31}$ We note that there is an experimental uncertainty of $20 \%-30 \%$ in the QE data due to possible variation of the polarization state.

${ }^{32}$ I. Horcas, R. Fernandez, J. M. Gomez-Rodriguez, J. Colchero, J. GomezHerrero, and A. M. Baro, Rev. Sci. Instrum. 78, 013705 (2007). 